\title{
ORIGINAL
}

\section{APORTACIÓN DE LA HISTORIA CLÍNICA DIGITAL A LA VIGILANCIA DE ENFERMEDADES DE DECLARACIÓN OBLIGATORIA}

\author{
María Ángeles Onieva-García (1), Begoña López-Hernández (1), María José Molina-Rueda (1), Natalia \\ Cabrera-Castro (1) y María del Mar Mochón-Ochoa (2).
}

(1) Unidad de Epidemiología. Distrito Granada-Metropolitano. UGC Interniveles de Salud Pública. Granada.

(2) Sección de Epidemiología. Servicio de Salud Pública. Delegación Territorial de Igualdad, Salud y Políticas Sociales. Granada.

\section{RESUMEN}

Fundamentos: En 2009 se implantó el sistema para la importación automática (IA) de casos con sospecha de enfermedad de declaración obligatoria (EDO) desde la historia clínica digital (HCD) a la RedAlerta, aplicación informática para la vigilancia epidemiológica en Andalucía. Hasta ahora, la contribución de este sistema a la clásica declaración activa no se ha determinado suficientemente. El principal objetivo de este estudio es evaluar la utilidad de IA en la provincia de Granada, entre 2009 y 2014

Métodos: Durante el periodo de estudio (2009-2014), un epidemiólogo validó si las EDO importadas satisfacían el criterio de declaración o no. Se halló la contribución de la IA a la RedAlerta y el porcentaje de validación de IA, estimando su IC $95 \%$.

Resultados: La contribución de la IA fue del 17,3\% (IC95\%: 16,118,5 ). Por tipo de declaración el 5,2\% (IC95\%:4,1-6,5) fueron las urgentes y $24,4 \%$ (IC95\%: 22,7-26,2) fueron ordinarias. La contribución fue superior al $45 \%$ en la enfermedad de Lyme, hipotiroidismo congénito, herpes genital, hepatitis C y otras hepatitis víricas. El 30\% (IC95\%:28,1-32) de las IA fueron validadas de las cuales el 39,9\% (IC95\%:33-47,2) fueron urgentes y el $29,1 \%$ (IC95\%:27,2-31,2\%) ordinarias. El porcentaje de validación fue superior al $45 \%$ (entre el 47,5 y el $100 \%$ ) en enfermedades vacunables, en las de transmisión sexual y en las de baja incidencia.

Conclusiones: Si bien no sustituye la declaración manual y requiere de un proceso de verificación, el sistema de incorporación automática es útil e incrementa la exhaustividad del sistema de vigilancia epidemiológica.

Palabras clave: Enfermedades de declaración obligatoria. Vigilancia epidemiológica. Sistemas de información. Validación.

\section{Correspondencia}

María Ángeles Onieva-García

UGC Interniveles de Salud Pública

Avda. Fuerzas Armadas, 2

18014 Granada, España.

mariaa.onieva@gmail.com

DOI:

\section{ABSTRACT \\ Contribution of Electronic Health Record in Surveillance of Notifiable Diseases}

Background: In 2009 a system was introduced for the automatic import (AI) of cases with suspected notifiable diseases (ND) from electronic medical record (EMR) to RedAlerta, an application for surveillance in Andalusia. At present, the contribution of this system to classical active statement has not been determined enough. The main objective of this study is to evaluate the usefulness of IA in the province of Granada, between 2009 and 2014.

Methods: During the study period (2009-2014), an epidemiologist assessed whether AI met declaration criteria or not. We calculate the contribution of AI to RedAlerta and the percentage of validation of AI, estimating 95\% CI.

Results: The contribution of AI was $17.3 \%$ (95\% CI 16.1 to 18.5$)$; and type of statement, $5.2 \%$ (95\% CI 4.1 to 6.5$)$ for urgent and $24.4 \%(95 \% \mathrm{CI}$ 22.7 to 26.2 ) for ordinary. The contribution was higher (more than $45 \%$ ) in Lyme disease, congenital hypothyroidism, genital herpes, hepatitis $\mathrm{C}$ and other viral hepatitis. $30 \%$ (95\% CI 28.1 to 32$)$ of AI were validated; $39.9 \%$ (95\% CI 33 to 47.2 ) urgent and $29.1 \%$ (95\% CI 27.2 to $31.2 \%$ ) ordinary. The percentage of validation was higher than $45 \%$ (between 47.5 and $100 \%$ ) in vaccine-preventable diseases, sexually transmitted infections and low incidence.

Conclusions: Although not replace manual reporting and requires verification, the AI system is useful and increases the completeness of the epidemiological surveillance system.

Key words: Notifiable diseases. Epidemiological monitoring. Information systems. Validation. 


\section{INTRODUCCIÓN}

La sospecha de enfermedades de declaración obligatoria (EDO) debe notificarse por los profesionales sanitarios del ámbito público y privado $^{1}$. Cada comunidad autónoma dispone de una relación propia de EDO. En Andalucía, la Orden de 11 de diciembre de 2008 establece la relación de EDO que se incluyen en el Sistema de Vigilancia Epidemiológica de Andalucía $(\mathrm{SVEA})^{2,3}$. La RedAlerta es la aplicación informática creada en 2002 para la vigilancia epidemiológica en Andalucía ${ }^{6,7}$.

El desarrollo de las tecnologías de la información facilita la identificación de EDO y complementa la declaración activa de los profesionales sanitarios ${ }^{4-6}$. En 2003 se implantó en Andalucía la historia clínica digital (HCD) denominada DIRAYA, una historia de salud única e inequívoca para cada persona atendida en el Sistema Sanitario Público de Andalucía. En 2009 se introdujo el sistema de importación automática (IA) de casos con sospecha de EDO desde la HCD a la RedAlerta. Este sistema de la IA se desarrolla actualmente de forma exclusiva en el ámbito de la atención primaria, verificándose a diario, desde el servicio de epidemiología, la idoneidad de la declaración importada, que valida o descarta cada caso $0^{6,7}$.

La precocidad en la declaración de EDO y la intervención temprana garantizan la efectividad de las medidas preventivas sobre contactos y/o medio $^{8,9}$. Existen dos tipos de declaración de EDO atendiendo a la necesidad (o no) de una actuación inmediata. La declaración de EDO ordinaria debe realizarse en 48 horas y la urgente en menos de 24 horas $^{2}$. En este sentido, se debe matizar que la IA tiene una demora promedio de 24 horas, por lo que las situaciones que requieran de intervención inmediata deberían comunicarse verbalmente.

Si bien desde 2009 coexiste el sistema tradicional de registro manual a la RedAlerta junto al sistema de IA, hasta el momento solo se han aportado datos preliminares de un único distrito de atención primaria para describir el valor añadido de este último en la declaración de EDO al Servicio de Vigilancia Epidemiológica de Andalucía (SVEA) ${ }^{6}$.

El objetivo de este estudio fue determinar la contribución y rentabilidad del sistema de importación automática de casos sospechosos de EDO desde la HCD a la RedAlerta en el ámbito provincial de Granada desde 2009 a 2014, con la finalidad de evaluar con mayor precisión su aportación a la vigilancia epidemiológica.

\section{MATERIAL Y MÉTODO}

Se diseñó un estudio transversal para determinar la utilidad de las IA de casos sospechosos de EDO producidas entre 2009 y 2014 correspondientes a la provincia de Granada.

El sistema de la IA se fundamenta en la selección de códigos CIE-9 considerados por consenso de expertos como pertinentes para la identificación de cada una de las EDO. De este modo, cada vez que se anota un diagnóstico con algunos de estos códigos en cualquiera de los apartados de juicio clínico, motivo de consulta o lista de problemas de la HCD de una persona, el caso sospechoso de EDO se importa como un registro pendiente de validación en la RedAlerta.

Desde 2009 hasta 2014, el epidemiólogo de atención primaria verificaba a diario, generalmente a primera hora de su jornada laboral, la idoneidad del caso importado. Para ello empleaba diferentes fuentes de confirmación: la HCD, la encuesta epidemiológica o la RedAlerta para la búsqueda de duplicados. Puesto que la IA tiene una demora de 24 horas, los casos verificados correspondían a la codificación de diagnósticos en la HCD del día previo.

Se consideraban como importaciones automáticas validadas las que satisfacían la definición de caso sospechoso, probable o confirmado para alguna $\mathrm{EDO}^{10}$, no estaban previamente registradas en la RedAlerta y eran oportunas en el tiempo (casos incidentes). 
Por otro lado, se clasificaban como importaciones automáticas no validadas las siguientes:

- Cuando no satisfacían la definición de caso sospechoso, probable o confirmado.

- Cuando satisfacían la definición de caso sospechoso, probable o confirmado pero su diagnóstico era pasado, es decir, con antecedentes de EDO pero sin enfermedad activa (por ejemplo, secuelas de poliomielitis). La validación de estos casos en la RedAlerta alteraría la incidencia real de enfermedad. El periodo límite consensuado para su exclusión fue la presencia de antecedentes de EDO de un año o más.

- Cuando se trataba de duplicados, es decir, EDO ya registradas en la RedAlerta. La comprobación de la duplicidad de casos se realiza mediante la introducción manual de identificadores inequívocos en la RedAlerta previamente a la validación del registro final, y es práctica habitual tanto en el proceso de verificación de la IA como en el proceso tradicional del registro manual.

- Cuando se trataba de errores de codificación en la HCD, por ejemplo, clasificación incorrecta del diagnóstico registrado en la historia.

Las variables de estudio fueron las que se describen a continuación:

- Tipo de EDO: cualquier enfermedad incluida en la relación de EDO que deben notificarse a través de la RedAlerta con al menos una IA desde la HCD.

- Tipo de declaración de EDO: urgente u ordinaria.

- Categorización de la IA: validadas o no validadas, según la definición previa.

- Año de declaración: año en el que se produce la IA y proceso de validación por el epidemiólogo. Permite analizar la evolución de la IA.

La principal fuente de información fue la RedAlerta, a partir de la cual se obtuvieron tanto las EDO procedentes de la IA de la HCD como las de registro manual.
Se procedió al cálculo de los siguientes indicadores:

- Porcentaje de contribución de la IA a la RedAerta: cociente entre el número de registros importados y validados, y el número total de EDO registradas en la RedAlerta.

- Porcentaje de validación de IA: cociente entre el número de registros importados y validados, $\mathrm{y}$ el número total de registros importados.

Para estimar la precisión de estos parámetros se procedió al cálculo de intervalos de confianza al 95\% (IC 95\%). Para el análisis temporal de la contribución y validación de la IA según año de declaración se emplearon modelos de regresión lineal con el cálculo de sus correspondientes coeficientes de correlación.

\section{RESULTADOS}

Desde 2009 a 2014 en la provincia de Granada se importaron automáticamente 2.128 registros sospechosos de EDO a la RedAlerta. El $100 \%$ de las IA se analizaron por un epidemiólogo, validándose un total de 639 (30,02 \%) registros sospechosos de EDO. Teniendo en cuenta que en el mismo periodo se declararon un total de 3.700 EDO a la RedAlerta, la contribución de la IA supuso el 17,3 \% (IC95 $\%$ : 16,1-18,5) de estas declaraciones. Por tipo de declaración, el 24,4 \% (IC95 \%: 22,7-26,2) fueron ordinarias (tabla 1) y el el 5,2\% (IC95 $\%$ : 4,1-6,5) fueron urgentes (tabla 2).

El $100 \%$ de los casos declarados de enfermedad de Lyme e hipotiroidismo congénito (no detectado en el Programa de Detección de Metabolapatías) procedieron de la IA. Otras EDO con un porcentaje destacable sobre el total fueron el herpes genital $(87,7 \%)$, la hepatitis C $(77 \%)$ y otras hepatitis víricas $(46,7$ \%) (tabla 1).

El $30 \%$ (IC95 \%: 28,1-32) de las cargas importadas desde la HCD fueron validadas. El 39,9 \% (IC95 \%: 33-47,2) fueron urgentes y el 29,1 \% (IC95 \%:27,2-31,2\%) ordinarias. Se descartó el $100 \%$ de los casos en las si- 


\begin{tabular}{|c|c|c|c|c|c|}
\hline \multicolumn{6}{|c|}{$\begin{array}{c}\text { Tabla } 1 \\
\text { Validación y contribución de la importación automática de } \\
\text { Enfermedades de Declaración Obligatoria en la provincia de Granada, 2009-2014 }\end{array}$} \\
\hline \multirow[t]{2}{*}{ 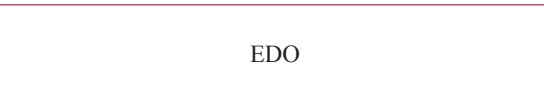 } & \multirow[b]{2}{*}{ Total } & \multicolumn{2}{|c|}{ Importaciones automáticas (IA) } & \multirow{2}{*}{$\begin{array}{c}\% \\
\text { Validación } \\
\text { de IA }\end{array}$} & \multirow{2}{*}{$\begin{array}{c}\% \\
\begin{array}{c}\text { Contribución } \\
\text { de IA }\end{array}\end{array}$} \\
\hline & & Validadas & No validadas & & \\
\hline \multicolumn{6}{|c|}{ Declaración ordinaria } \\
\hline Aspergilosis & 27 & 2 & 3 & 40 & 7,4 \\
\hline Carbunco & 0 & 0 & 3 & 0 & 0 \\
\hline Criptosporidiosis & 16 & 7 & 3 & 70 & 43,8 \\
\hline Disentería & 8 & 1 & 0 & 100 & 12,5 \\
\hline Encefalopatías Espongiformes Transmisibles Humanas & 3 & 0 & 1 & 0 & 0 \\
\hline Enfermedad de Lyme & 1 & 1 & 3 & 25 & 100 \\
\hline Fiebre exantemática mediterránea & 52 & 11 & 54 & 16,9 & 21,2 \\
\hline Fiebre Q & 16 & 4 & 5 & 44,4 & 25 \\
\hline Fiebre tifoidea y paratifoidea & 2 & 0 & 3 & 0 & 0 \\
\hline Hepatitis B & 56 & 16 & 98 & 14 & 28,6 \\
\hline Hepatitis C & 239 & 184 & 576 & 24,2 & 77 \\
\hline Hepatitis vírica, otras & 15 & 7 & 51 & 12,1 & 46,7 \\
\hline Herpes genital & 114 & 100 & 47 & 68 & 87,7 \\
\hline Infección genital por Clamydia trachomatis & 29 & 2 & 0 & 100 & 6,9 \\
\hline Infección gonocócica & 199 & 48 & 12 & 80 & 24,1 \\
\hline Intoxicación aguda por plaguicidas & 7 & 3 & 1 & 75 & 42,9 \\
\hline Leishmaniasis & 36 & 3 & 19 & 13,6 & 8,3 \\
\hline Lepra & 3 & 0 & 1 & 0 & 0 \\
\hline Paludismo & 35 & 1 & 0 & 100 & 2,9 \\
\hline Parotiditis & 237 & 76 & 114 & 40 & 32,1 \\
\hline Sífilis & 390 & 19 & 55 & 25,7 & 4,9 \\
\hline Sífilis congénita & 4 & 0 & 1 & 0 & 0 \\
\hline Tos ferina & 253 & 57 & 63 & 47,5 & 22,5 \\
\hline Tuberculosis & 582 & 26 & 269 & 8,8 & 4,5 \\
\hline Total & 2.324 & 568 & 1.382 & 29,1 & 24,4 \\
\hline
\end{tabular}

guientes enfermedades: carbunco, encefalopatías espongiformes transmisibles humanas (EETH), fiebre tifoidea y paratifoidea, lepra, sífilis congénita, cólera, fiebre amarilla, listeriosis, parálisis fláccida, poliomielitis, reacción postvacunal grave y rubéola congénita (tablas 1 y 2). De los 27 tipos de EDO para los que hubo alguna IA, 12 tuvieron un porcentaje de validación correcta superior al $45 \%$ (entre el 47,5 y el $100 \%$ ) como enfermedades vacunables (tos ferina y sarampión), enfermedades de transmisión sexual (herpes genital, infección gonocócica e infección genital por Clamydia trachomatis), enfermedades de baja incidencia (brucelosis) o infra-diagnosticadas (criptosporidiosis), así como las meningitis (víricas y otras de origen infeccioso), que requieren de intervención urgente (tablas 1 y 2).

En el análisis temporal según año de declaración se observó una tendencia creciente en la contribución $\left(\mathrm{R}^{2}=0,59\right.$; coeficiente $\left.=4,14\right)$ y decreciente para la validación de la IA $\left(R^{2}=0,95\right.$; coeficiente $\left.=-4,18\right)$. La contribución de la IA aumentó cada año, a excepción del descenso en 2011 (10,1\%; IC95 \%: 8,3-12,3) y $2014(25,9 \%$; IC95 \%: 22,7-29,4), alcanzando el mayor porcentaje [32,1 \% (IC95 \%: $28,5-35,8)$ ] en 2013. Al contrario, la validación disminuyó en el sub-periodo 2009-2012, 


\begin{tabular}{|c|c|c|c|c|c|}
\hline \multicolumn{6}{|c|}{$\begin{array}{c}\text { Tabla } 2 \\
\text { Validación y contribución de la importación automática de } \\
\text { Enfermedades de Declaración Obligatoria en la provincia de Granada, 2009-2014 }\end{array}$} \\
\hline \multirow{2}{*}{ 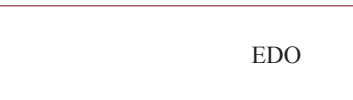 } & \multirow[b]{2}{*}{ Total } & \multicolumn{2}{|c|}{ Importaciones automáticas (IA) } & \multirow{2}{*}{$\begin{array}{c}\% \\
\text { Validación } \\
\text { de IA }\end{array}$} & \multirow{2}{*}{$\begin{array}{c}\% \\
\text { Contribución } \\
\text { de IA }\end{array}$} \\
\hline & & Validadas & No validadas & & \\
\hline \multicolumn{6}{|c|}{ Declaración urgente } \\
\hline Brucelosis & 65 & 12 & 10 & 54,5 & 18,5 \\
\hline Cólera & 0 & 0 & 1 & 0 & 0 \\
\hline Enfermedad neumocócica invasora & 311 & 1 & 4 & 20 & 0,3 \\
\hline Fiebre amarilla & 0 & 0 & 1 & 0 & 0 \\
\hline Hepatitis A & 102 & 5 & 31 & 13,9 & 4,9 \\
\hline Hipotiroidismo congénito & 3 & 3 & 25 & 10,7 & 100 \\
\hline Listeriosis & 72 & 0 & 1 & 0 & 0 \\
\hline Meningitis bacteriana, otras & 70 & 8 & 12 & 40 & 11,4 \\
\hline Meningitis infecciosas, otras & 12 & 2 & 1 & 66,7 & 16,7 \\
\hline Meningitis víricas & 397 & 8 & 1 & 88,9 & 2 \\
\hline Parálisis fláccida & 6 & 0 & 1 & 0 & 0 \\
\hline Poliomielitis & 0 & 0 & 10 & 0 & 0 \\
\hline Reacción postvacunal grave & 3 & 0 & 1 & 0 & 0 \\
\hline Rubeola congénita & 1 & 0 & 2 & 0 & 0 \\
\hline Sarampión & 334 & 32 & 6 & 84,2 & 9,6 \\
\hline Total & 1.376 & 71 & 107 & 39,9 & 5,2 \\
\hline
\end{tabular}

Figura 1

Evolución de la validación de la importación automática de Enfermedades de Declaración Obligatoria en la provincia de Granada, 2009-2014

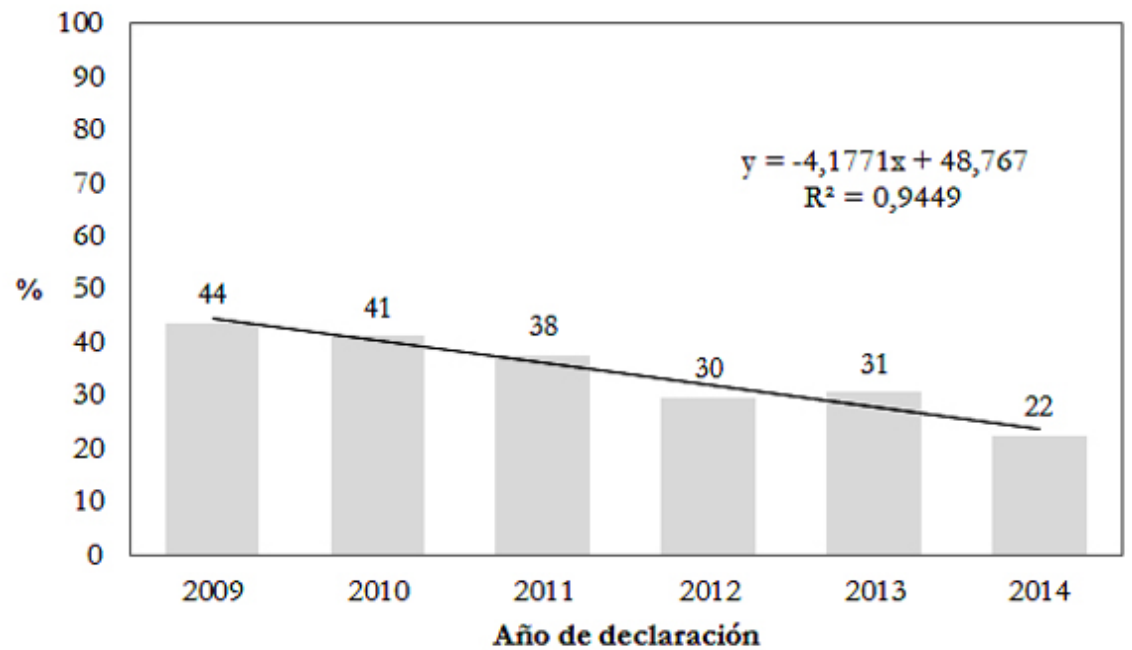


Figura 2

Evolución de la contribución de la importación automática de Enfermedades de Declaración Obligatoria en la provincia de Granada, 2009-2014

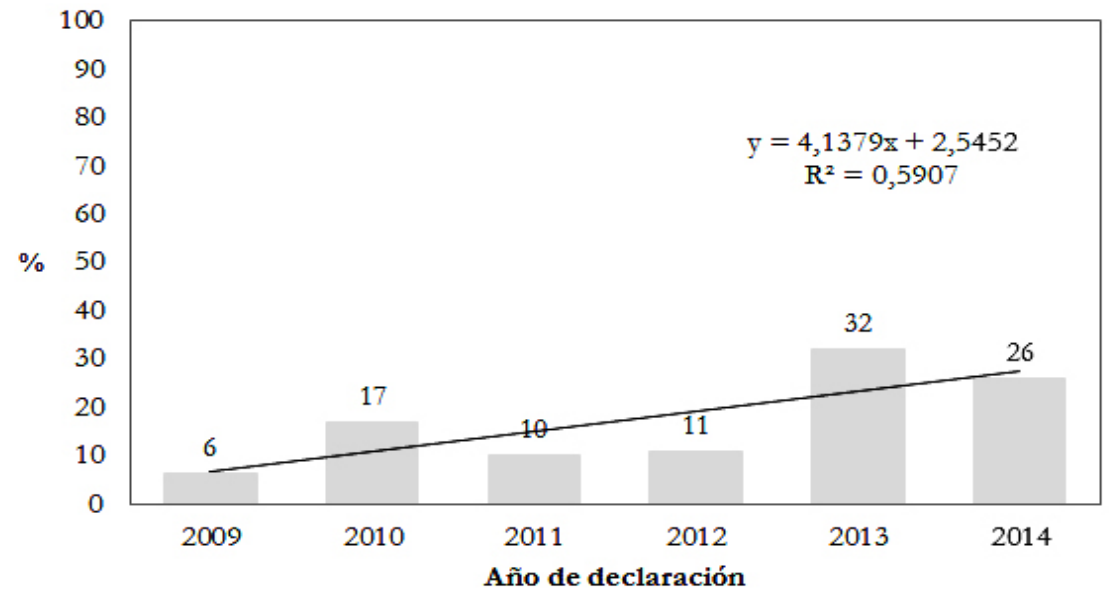

pasando del 43,7\% (IC95 \%: 32,8-55,2) en 2009 al 29,6\% (IC95 \%: 23,2-36,9) en 2012, ascendiendo discretamente en 2013 (30,5\%; IC95\%: 27,1-34,1) y descendiendo nuevamente en 2014 (22,4\%; IC95 \%: 19,625,5) (figuras 1 y 2).

\section{DISCUSIÓN}

El sistema de la importanción automática de EDO desde la HCD nos ha aproximado al escenario real de la incidencia de estas enfermedades en la población. La implantación de la IA, como fuente de información adicional a las ya existentes, ha contribuido al SVEA sumando un número de casos considerable de EDO durante el periodo de estudio, y por ende, disminuyendo la infradeclaración que afecta a los sistemas de vigilancia epidemiológica ${ }^{8,9}$. Aunque la demora implícita en la importación resta utilidad en el caso de EDO que requieren de intervención urgente, cabe destacar que algunas de estas enfermedades se declararon mediante IA, acortando tiempos de intervención que serían mayores al notificarse y/o declararse a posteriori por medio de otras fuentes de información.
La incorporación de este sistema ha contribuido de manera especial en la vigilancia epidemiológica de las EDO que no llegan a confirmarse microbiológicamente. En Andalucía, la infradeclaración derivada de la escasa notificación activa por parte de los profesionales sanitarios resulta atenuada gracias a la comunicación diaria entre los servicios de microbiología y de medicina preventiva de los resultados de laboratorio que pudieran abocar en una EDO. Sin embargo, algunas EDO se caracterizan por su baja oportunidad de confirmación microbiológica (por ejemplo, en los casos de herpes genital no suelen solicitarse pruebas de confirmación) mientras que otras EDO no requieren de pruebas microbiológicas, confirmándose únicamente con criterios epidemiológicos. Este tipo de EDO, por tanto, no podrían declararse a través de la información proporcionada por los laboratorios a los servicios declarantes. Así, la importación automática suma oportunidades de declaración de EDO que escapan de los sistemas tradicionales (en nuestro estudio, 9 de cada 10 casos de herpes genital procedieron de la carga automática). Además, en el caso de la EDO que no se confirma microbioló- 
gicamente y que además precisa de intervenciones comunitarias (medidas de aislamiento, estudio de contactos o quimioprofilaxis) la aportación del sistema de la IA se traduce en seguridad poblacional ${ }^{16}$. Esto es lo que ocurre con enfermedades prevenibles mediante vacunación (tos ferina,...). En el caso de la tuberculosis (TB), dada la trascendencia sanitaria y social de esta enfermedad, si bien la contribución y validación de la IA fue baja la detección de un solo caso sospechoso de TB, especialmente pulmonar o TB infantil, podría evitar la aparición de casos secundarios, rentabilizando el coste de la verificación de las IA no validadas.

Para estimar la rentabilidad de este sistema evaluamos el porcentaje de validación de la IA. Durante el periodo de estudio, un tercio de las importaciones a la RedAlerta fueron validadas, mientras que los dos tercios restantes no lo fueron, en su mayoría por errores, duplicidades o antecedentes de EDO sin enfermedad activa. En este sentido es reseñable el caso de la hepatitis $C$, de la que se descartaron hasta 576 registros importados. Esta enfermedad evoluciona hacia la cronicidad derivando en IA reiteradas desde la $\mathrm{HCD}$, tantas como consultas de seguimiento en atención primaria en las que los profesionales médicos codifican esta patología en el diagnóstico al alta. Esta circunstancia, además de suponer un trabajo estéril para el epidemiólogo, puede provocar una sobreestimación de la frecuencia de la enfermedad. Puesto que es requisito la confirmación de laboratorio para la declaración de un caso nuevo de hepatitis $\mathrm{C}$, sería recomendable excluir esta EDO del sistema de la IA, filtrando la información a través de los laboratorios de microbiología y verificando a posteriori, a través de la HCD u otras pruebas de laboratorio anteriores, la idoneidad de su registro manual.

En nuestro análisis temporal hallamos un aumento en la contribución de la IA a la RedAlerta y una disminución progresiva de la validación de la IA a lo largo del periodo de estudio. Este fenómeno se explica por la prác- tica creciente de codificación diagnóstica por parte del personal de atención primaria que, al tiempo que suma declaraciones al total de EDO registradas en el sistema de vigilancia epidemiológica, también aumenta la carga de posibles duplicados o codificaciones erróneas, con la consiguiente disminución de la rentabilidad o validación de la IA.

Se estima que el proceso de verificación supone un $10 \%$ de la jornada laboral de un epidemiólogo. Aunque a priori podría deducirse que este proceso deriva en costes indirectos elevados, principalmente por el número de importaciones no validadas, consideramos, en base a nuestros resultados, que la utilidad del sistema de la IA queda demostrada. No obstante, la incorporación de un procedimiento computarizado de depuración de duplicados contribuiría enormemente a la eficiencia de este sistema.

A la luz de los resultados puede concluirse que, si bien la importación automática no sustituye a la declaración activa y/o urgente de algunas EDO, sí complementa los casos registrados en el sistema de vigilancia epidemiológica actual. Consideramos que toda aportación a la vigilancia epidemiológica es un avance irrenunciable y subrayamos la utilidad de la integración de este sistema (u otros similares) junto a los sistemas tradicionales en la práctica habitual de la notificación activa de EDO.

\section{BIBLIOGRAFÍA}

1. Boletín Oficial del Estado. Real Decreto 2210/1995, de 28 de diciembre, por el que se crea la red nacional de vigilancia epidemiológica. BOE núm 21 de 24/1/1996.

2. Boletín Oficial de la Junta de Andalucía. Orden de 11 de diciembre de 2008, por la que se modifica la Orden de 19 de diciembre de 1996, por la que se desarrolla el Sistema de Vigilancia Epidemiológica en la Comunidad Autónoma de Andalucía y se establece la relación de enfermedades de declaración obligatoria. BOJA núm 4 de 8/1/2009.

3. Boletín Oficial de la Junta de Andalucía. Decreto 66/1996, de 13 de febrero, por el que se constituye, en la Comunidad Autónoma de Andalucía, el Sistema de Vigilancia Epidemiológica y se determinan normas sobre el mismo. BOJA núm 35 de 19/3/1996. 
4. Ward M, Brandsema P, van Straten E, et al. Electronic reporting improves timeliness and completeness in infectious disease notification. The Netherlands, 2003. Euro Surveill. 2005;10:27-30.

5. Rolfhamre P, Jansson A, Arneborn M, et al. SmiNet-2: description of an internetbased surveillance system for communicable diseases in Sweden. Euro Surveill. 2006;11:103-7.

6. Molina MJ, Cabrera N, Onieva MA, López B. Historia de salud digital (Diraya): un recurso en la vigilancia epidemiológica. Gac Sanit. 2014;28(4):341-2.

7. Valencia R, Román E, García-León FJ, et al. Sistema de alerta: una prioridad en vigilancia epidemiológica. Gac Sanit. 2003;17:520-2.

8. Rubio-Cirilo L, Martín-Ríos MD, Casas-Cámara G, et al. Enfermedades de declaración obligatoria: conocimiento y notificación en el ámbito hospitalario. Enferm Infecc Microbiol Clin. 2013; 31:643-8.

9. Turnberg W, Daniell W, Duchin J. Notificable infectious disease reporting awareness among physicians and registers nurses in primary care and emergency department settings. Am J Infect Control. 2010;38:410-2.

10. Junta de Andalucía. Consejería de Igualdad, Salud y Políticas Sociales [Internet]. Sevilla (Andalucía): Consejería de Igualdad, Salud y Políticas Sociales; 2012. Red Alerta: Protocolos. Procedimientos; 2012 [citado 2014-11-01]. Disponible en: http:/www.juntadeandalucia.es/salud/channels/temas/temas es/P 4 SALUD PUBLICA/P 1 VIGILANCIA DE LA SALUD/red_alerta/red_alerta?perfil $=$ org\&desplegar $=/$ temas_es/P 4_SALUD_PUBLICA/\&idioma $=$ es\&tema=/ temas_es/P 4 SALUD_PUBLICA/P 1 VIGILANCIA DE LA SATLUD/red alerta/\&contenido=/channels/temas/temas_es/P_4_SALUD_PUBLICA/P_1_VIGILANCIA_DE_LA_SĀĒUD/red_alerta/red_alerta 